\title{
Short Communication: \\ Identification of culturable marine fungi and bacteria from coastal region in Brunei Darussalam
}

\author{
HUSSEIN TAHA, POOJA SHIVANAND", MOHAMAD ABDUL AZIM ZAINUDIN, \\ NURUL AZIRAH HADANAN \\ Environmental and Life Sciences Program, Faculty of Science, Universiti Brunei Darussalam. J1. Tungku Link BE1410, Brunei Darussalam. \\ vemail: pooja.shivanand@ubd.edu.bn
}

Manuscript received: 6 January 2020. Revision accepted: 16 February 2021.

\begin{abstract}
Taha H, Shivanand P, Zainudin MAA, Hadanan NA. 2021. Short Communication: Identification of culturable marine fungi and bacteria from coastal region in Brunei Darussalam. Biodiversitas 22: 1326-1331. Microbial diversity in Brunei Darussalam located on northwest coast of Borneo is not well explored. This study aimed to isolate and identify culturable marine fungi and bacteria from coastal regions. Microbial identification was carried out via DNA barcoding using rRNA-ITS marker for fungi and 16S rRNA marker for bacteria. Nine marine fungal isolates were identified to five genera as Aspergillus, Penicillium, Phialemoniopsis, Purpureocillium and Trametes. However, only one genus, Pseudoalteromonas was identified from ten marine bacterial isolates with at least three different Pseudoalteromonas species expected from the isolation. This study provided an insight into the diversity of culturable marine microbes from a coastal ecosystem. As the microbes were found culturable and can easily grow under saline conditions, they are potential of considerable biotechnological interest.
\end{abstract}

Keywords: Beach ecosystem, cultivable microbes, DNA barcoding, marine microbes, microbial diversity

\section{INTRODUCTION}

Marine environments which consist of the coastal and deep-sea regions are a great reservoir of microbial diversity including various fungi (Manohar et al. 2013; Yakop et al. 2019) and bacteria (Das et al. 2006). Marine environments are also exceptionally complex and have unique conditions of high salinity. In response, many marine microbes have evolved unique features and metabolites to adapt to such unique conditions. The isolation of marine microbes and their bioactive metabolites including fungi as potential sources of cosmeceuticals (Agrawal et al. 2018) and anticancer compounds (Deshmukh et al. 2018) is an active area of research (Zhang et al. 2005; Carroll et al. 2019). Recent studies on the microbial diversity of different habitats in Brunei Darussalam had found a diversity of culturable fungi and bacteria of potential biotechnological values (Taha et al. 2020a; Taha et al. 2020b). However, the microbial diversity of coastal region in Brunei Darussalam has not been well explored. Coastal ecosystems generally are extremely dynamic and highly productive due to eutrophication (Danovaro and Pusceddu 2007). Therefore, it is of considerable biotechnological interest to explore such habitat. It is also of ecological interest as it would increase our understanding of the marine ecosystem. Furthermore, the molecular diversity of fungi from the marine habitats has been reported mostly from the deep-sea regions but only a few from the coastal regions (Manohar et al. 2013). Therefore, this present study was aimed to isolate and identify culturable marine fungi and bacteria from a beach ecosystem in Brunei Darussalam.

\section{MATERIALS AND METHODS}

\section{Sample collection}

The study area of present study Berakas Beach (459'39.12" N 11455'13.08" E) was located in Berakas Forest Reserve Recreational Park in Brunei Darussalam, northwest coast of Borneo, Southeast Asia. Seawater samples were collected from few sites, $0-5 \mathrm{~cm}$ below the water surface and $2-5 \mathrm{~m}$ from the shoreline. The temperature, $\mathrm{pH}$, and salinity of the seawater samples were measured during collection to be $32{ }^{\circ} \mathrm{C}, 8.2$, and 2.5-3.00 $\%$, respectively.

\section{Isolation of microbes}

For fungal isolation, potato dextrose agar (PDA; Oxoid, UK) plates were prepared aseptically using synthetic seawater medium ( $\mathrm{pH} 8.2 ; 28.13 \mathrm{~g} / \mathrm{L}$ sodium chloride, 0.77 $\mathrm{g} / \mathrm{L}$ potassium chloride, $1.60 \mathrm{~g} / \mathrm{L}$ calcium chloride dihydrate, $4.80 \mathrm{~g} / \mathrm{L}$ magnesium chloride 6-hydrate, 0.11 $\mathrm{g} / \mathrm{L}$ sodium hydrogen carbonate and $3.50 \mathrm{~g} / \mathrm{L}$ magnesium sulfate 7-hydrate). The synthetic seawater was used in order to isolate fungi that could tolerate high salinity. Chloramphenicol was added at $25 \mu \mathrm{g} / \mathrm{mL}$ to the PDA plates to inhibit bacterial growth. For bacterial isolation, nutrient agar (NA; Oxoid, UK) plates were prepared using the synthetic seawater medium in order to also isolate 
halotolerant bacteria. Each of the collected seawater samples was ten-fold serially diluted using $3 \%$ (w/v) sterile saline solution, producing $10^{-1}, 10^{-2}$ and $10^{-3}$ dilutions. The PDA and NA plates were inoculated with $100 \mu \mathrm{L}$ of each seawater sample and all the dilutions. All plates were incubated at $25 \pm 2^{\circ} \mathrm{C}$ for fungi and $37 \pm 2^{\circ} \mathrm{C}$ for bacteria until microbial growth was observed. Microbial colonies were selected based partly on their different morphological characteristics. For fungi, this involved macroscopic observation of the colony morphology on the agar plates, and microscopic observation of the cells based on Barnett and Hunter (1998). While for bacteria, observations of colony characteristics and cellular morphology after Gram staining were carried out based on Leboffe and Pierce (2012). The selected colonies were subcultured several times via streaking in order to obtain pure cultures. A total of 9 fungal and 10 bacterial isolates were used for DNA barcoding. The isolates were coded alphanumerically with the codes, UBDFC and UBDBC for fungal and bacterial isolates, respectively.

\section{Microbial identification}

Isolates were identified via Biolog and DNA barcoding. Biolog identification was carried out as previously described (Taha et al. 2020a). For DNA barcoding, genomic DNA was extracted using Quick-DNA Fungal/Bacterial Miniprep Kit (Zymo Research, USA) following the manufacturer's instructions. The DNA was barcoded using rRNA-ITS sequence ( 700 bp) for fungi and 16S rRNA sequence ( 1400 bp) for bacteria. The resulting DNA sequences were deposited in the GenBank database with the accession numbers shown in Tables 1 and 2. The DNA sequences were compared using BLAST with the available sequences in the NCBI Nucleotide Collection (nr/nt) Database and the NCBI 16S ribosomal RNA sequences Database for fungi and bacteria, respectively. Nearest BLAST matches with high identity and max score values were selected. To align DNA sequences and construct phylogenetic tree, MEGA7 was used (Kumar et al. 2016). The tree was constructed via the neighbor-joining method and the evolutionary distance was calculated via the Kimura 2-parameter method.

\section{RESULTS AND DISCUSSION}

Biolog identification did not result in any significant match (similarity index was less than 0.5 ). Thus, only the results from DNA barcoding were used in this study. All 9 fungal isolates were identified by DNA barcoding to the genus level (Table 1; Fig. 1). Two isolates (UBDFC07 and UBDFC10) were $100 \%$ identical to both Aspergillus nomius and Aspergillus pseudonomius. Thus, these isolates were identified only as Aspergillus species. Similarly, isolate UBDFC01 was $100 \%$ identical to both Penicillium citreosulfuratum and Penicillium citreonigrum. Thus, it was identified only as Penicillium species. Two isolates (UBDFC04 and UBDFC05) were highly identical to 3 species under the class Sordariomycetes: Phialemoniopsis pluriloculosa (99 \%; max score 960), Sarocladium strictum (98 \%; $\max$ score 940) and Phialemonium aff. dimorphosporum (99\%; max score 939). Thus, the two isolates were identified as Phialemoniopsis species due to its highest max score. Two isolates (UBDFC02 and UBDFC09) were $100 \%$ identical to Purpureocillium lilacinum (synonym Paecilomyces lilacinus) and thus, they were identified as Purpureocillium species. Two isolates (UBDFC03 and UBDFC08) were $99 \%$ identical to 2 species under the class Agaricomycetes: Trametes polyzona (synonym Coriolopsis polyzona; max score 1128) and Tricholoma robustum (max score 1108). As the max score was the highest, the isolates were identified as Trametes species. In total, 5 fungal genera were identified from 9 culturable marine isolates.

All 10 bacterial isolates were identified by DNA barcoding to one genus, Pseudoalteromonas (Table 2; Fig. 2). Two isolates (UBDBC01 and UBDBC05) were $99 \%$ identical to several species of Pseudoalteromonas but the highest max score was obtained from $P$. gelatinilytica. Thus, they were only identified as Pseudoalteromonas species. Three isolates were also $99 \%$ identical with several species of Pseudoalteromonas with P. piscicida showing the highest max score. Thus, they also identified as Pseudoalteromonas species. Similarly, 5 isolates (UBDBC08, UBDBC11, UBDBC12, UBDBC13, and UBDBC14) were also $99 \%$ identical to several species with the highest max score obtained from $P$. shioyasakiensis although it did not form sister groups with the 5 isolates in the phylogenetic tree. Thus, they were only identified as Pseudoalteromonas species. Although this study only identified 1 bacterial genus, at least 3 different species were expected from the 10 culturable marine isolates.

The present study isolated and identified five genera of culturable fungi from the coastal region in Brunei Darussalam as Aspergillus, Penicillium, Phialemoniopsis, Purpureocillium, and Trametes. This seems to indicate that the coastal region is diverse in its fungal community. Similarly, a previous study on the diversity of fungi in the sandy beach soil of Pulau Pinang, Malaysia also found the sandy beach was diverse with seven fungal genera identified including Aspergillus and Penicillium (Zakaria et al. 2011). Although BLAST results showed 99 to $100 \%$ similarity, this study could only identify the isolates up to the genus level only. A second DNA marker or other relevant methods could be used to confirm the isolates to the species level. For example, beta-tubulin marker was used in addition to rRNA-ITS marker for the identification of Penicillium species (Gonçalves et al. 2019). Biolog could not confirm the microbial identification due to limited entries in the Biolog databases. 
Table 1. Culturable marine fungi isolated from Brunei Darussalam

\begin{tabular}{llcll}
\hline Isolate ID & \multicolumn{1}{c}{ Identification } & $\begin{array}{c}\text { GenBank } \\
\text { accession no. }\end{array}$ & \multicolumn{1}{c}{ Top BLAST match } & $\begin{array}{c}\text { Identity } \\
(\%)\end{array}$ \\
\hline UBDFC07 & Aspergillus sp. & MK116442 & Aspergillus nomius \& Aspergillus pseudonomius & 100 \\
UBDFC10 & Aspergillus sp. & MK116445 & Aspergillus nomius \& Aspergillus pseudonomius & 100 \\
UBDFC01 & Penicillium sp. & MK116437 & Penicillium citreosulfuratum \& Penicillium citreonigrum & 100 \\
UBDFC04 & Phialemoniopsis sp. & MK116440 & Phialemoniopsis pluriloculosa & 99 \\
UBDFC05 & Phialemoniopsis sp. & MK116441 & Phialemoniopsis pluriloculosa & 99 \\
UBDFC02 & Purpureocillium sp. & MK116438 & Purpureocillium lilacinum & 100 \\
UBDFC09 & Purpureocillium sp. & MK116444 & Purpureocillium lilacinum & 100 \\
UBDFC03 & Trametes sp. & MK116439 & Trametes polyzona & 99 \\
UBDFC08 & Trametes sp. & MK116443 & Trametes polyzona & 99 \\
\hline
\end{tabular}

Table 2. Culturable marine bacteria isolated from Brunei Darussalam.

\begin{tabular}{llcll}
\hline Isolate ID & Identification & GenBank accession no. & Top BLAST match & Identity (\%) \\
\hline UBDBC01 & Pseudoalteromonas sp. & MK101084 & Pseudoalteromonas gelatinilytica & 99 \\
UBDBC05 & Pseudoalteromonas sp. & MK101085 & Pseudoalteromonas gelatinilytica & 99 \\
UBDBC07 & Pseudoalteromonas sp. & MK101086 & Pseudoalteromonas piscicida & 99 \\
UBDBC09 & Pseudoalteromonas sp. & MK101088 & Pseudoalteromonas piscicida & 99 \\
UBDBC10 & Pseudoalteromonas sp. & MK101089 & Pseudoalteromonas piscicida & 99 \\
UBDBC08 & Pseudoalteromonas sp. & MK101087 & Pseudoalteromonas shioyasakiensis & 99 \\
UBDBC11 & Pseudoalteromonas sp. & MK101090 & Pseudoalteromonas shioyasakiensis & 99 \\
UBDBC12 & Pseudoalteromonas sp. & MK101091 & Pseudoalteromonas shioyasakiensis & 99 \\
UBDBC13 & Pseudoalteromonas sp. & MK101092 & Pseudoalteromonas shioyasakiensis & 99 \\
UBDBC14 & Pseudoalteromonas sp. & MK101093 & Pseudoalteromonas shioyasakiensis & 99 \\
\hline
\end{tabular}

The biotechnological potentials of the fungal isolates in the present study are yet to be investigated. Nevertheless, a previous study also isolated Aspergillus species from the coastal waters of Southern China, which showed production of extracellular enzymes and pelletization capability (Li et al. 2014). Aspergillus species were also isolated from the Mediterranean sponge collected from the coast of Israel, which produced novel terpenoids (Cohen et al. 2011). Aspergillus species were also isolated from the marine sediments of South Indian Coast and showed antibacterial activity (Mathan et al. 2011).

Although one isolate of Penicillium species was identified in the present study, many Penicillium species including one novel species were isolated in a recent study from the coastal marine environments in Portugal (Gonçalves et al. 2019). This indicates that coastal habitat can be a good reservoir of this genus of fungi. Penicillium strains were also the most frequently isolated fungi from the sandy soil of Egyptian beaches (Migahed 2003). Penicillium strains were also isolated from the eastern coast of Alexandria in Egypt with one strain showing antibacterial, antifouling and anticancer activities (Amer et al. 2019).

Phialemoniopsis is a new genus of fungi under the class Sordariomycetes, which includes the reclassification of Phialemonium curvatum as Phialemoniopsis curvata (Perdomo et al. 2013). Not much has been reported on the isolation of Phialemoniopsis from marine environments. However, Phialemoniopsis species isolated from the estuarine mangrove sediment of Indian Sundarban were reported to have application in the biosynthesis of iron oxide nanoparticles (Mahanty et al. 2019). Production of secondary metabolites from Phialemoniopsis species isolated from the island of Hawaii had also been reported (Kaur et al. 2014).

The genus Purpureocillium had been previously reported from marine environments such as $P$. lilacinum isolated from halophytic plants from the west coast of Korea (Khalmuratova et al. 2015). Interestingly, $P$. lilacinum had also been found persistently in the digestive system of brown shrimp, suggesting the possibility of symbiotic relationship between the fungus and shrimp (Siegenthaler et al. 2019). A marine isolate with $99 \%$ similarity with $P$. lilacinum was also isolated from a jellyfish and showed antimicrobial activity (Yue et al. 2015).

The genus Trametes had been previously revised by considering a number of genera as synonyms of Trametes (Justo and Hibbett 2011). There is little report on the isolation of Trametes species from the coastal habitats. However, $T$. versicolor and other fungi were reported from the deep-subseafloor sediments in the Canterbury Basin in New Zealand, highlighting the diversity of fungi in the marine environments (Rédou et al. 2015). From biotechnological perspective, several Trametes species from Serbia had been shown to have significant medicinal potentials (Knežević et al. 2018). 


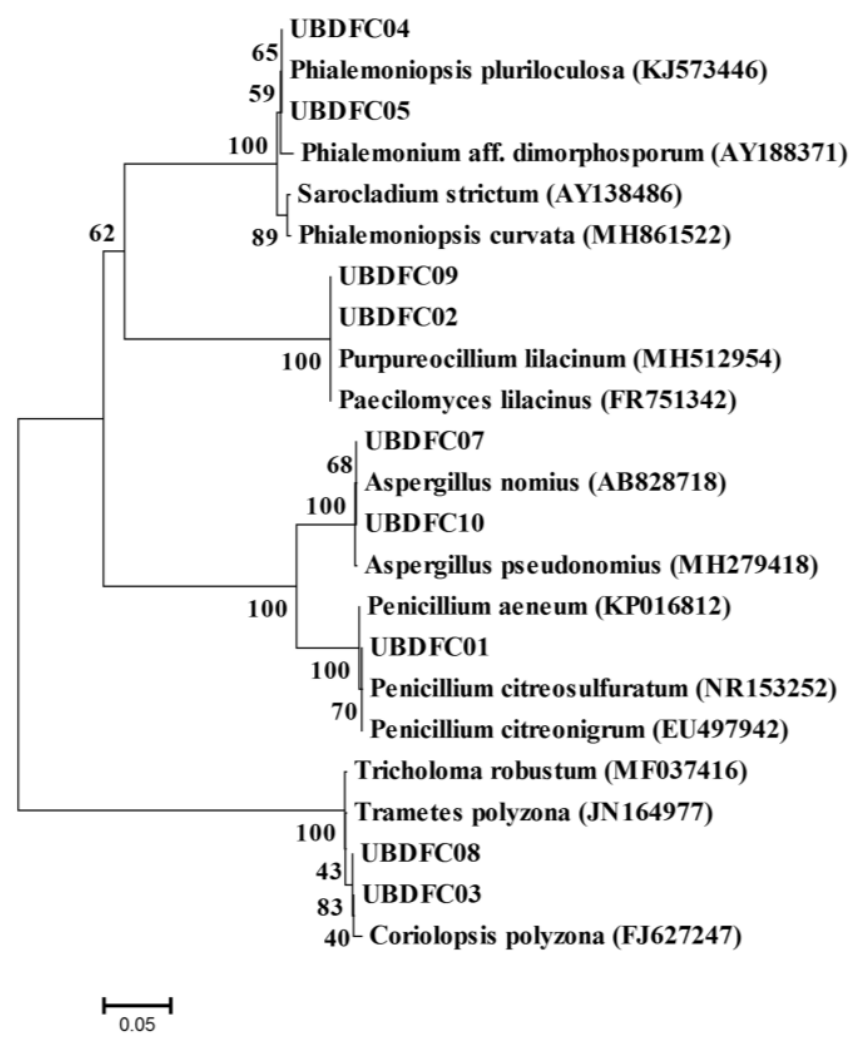

Figure 1. Phylogenetic tree of fungi based on rRNA-ITS sequences. Numbers at nodes represent bootstrap percentages based on 1000 replicates. GenBank accession number is shown in bracket. Scale refers to evolutionary distance in the unit of no. of base substitutions per site.

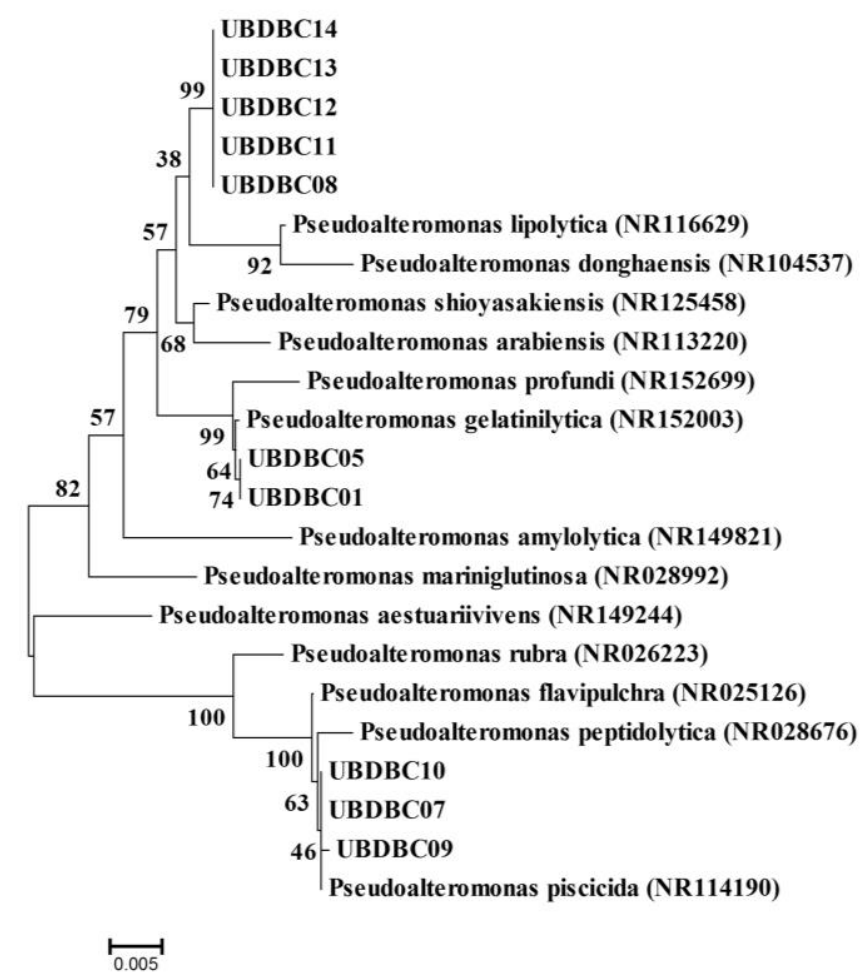

Figure 2. Phylogenetic tree of bacteria based on 16S rRNA sequences. Numbers at nodes represent bootstrap percentages based on 1000 replicates. GenBank accession number is shown in bracket. Scale refers to evolutionary distance in the unit of no. of base substitutions per site 
The present study only isolated and identified one genus of culturable bacteria from the coastal region in Brunei Darussalam i.e. Pseudoalteromonas. However, at least three different Pseudoalteromonas species were expected from the isolation. Pseudoalteromonas species are widespread in marine environments like coastal seawater of Delaware Bay (Richards et al. 2019), the Antarctic coastal seawater and the deep-sea sediment near the Okinawa Trough (Qin et al. 2011). This bacterial genus has attracted significant interest because many species have been shown interesting biological activities (such as antibacterial and antifouling) and produce extracellular enzymes, toxins and polysaccharides (Holmström and Kjelleberg 1999).

In conclusion, the present study through DNA barcoding identified five genera of culturable marine fungi like Aspergillus, Penicillium, Phialemoniopsis, Purpureocillium and Trametes, and identified one genus of culturable marine bacteria, Pseudoalteromonas from the coastal region of Brunei Darussalam. This seems to suggest that the coastal environment had diverse fungal community. The culturable and salt-tolerant microbes are potential sources of biotechnological products and applications that are yet to be investigated.

\section{ACKNOWLEDGEMENTS}

This work was funded by Universiti Brunei Darussalam under a research grant no. UBD/OAVCRI/CRGWG (010)/170501. The authors thank Crop Protection Unit, Department of Agriculture and Agrifood, Ministry of Primary Resources and Tourism of Brunei Darussalam for their biologist identification assistance.

\section{REFERENCES}

Agrawal S, Adholeya A, Barrow CJ, Deshmukh SK. 2018. Marine fungi: an untapped bioresource for future cosmeceuticals. Phytochem Lett 23: 15-20. DOI: 10.1016/j.phytol.2017.11.003.

Amer MS, Ellatif HHA, Hassan SWM, Aboelela GM, Gad AM. 2019. Characterization of some fungal strains isolated from the Eastern coast of Alexandria, Egypt, and some applications of Penicillium crustosum. Egypt J Aquat Res 45 (3): 211-217. DOI: 10.1016/j.ejar.2019.06.006.

Barnett HL, Hunter BB. 1998. Illustrated Genera of Imperfect Fungi $\left(4^{\text {th }}\right.$ edition). APS Press, St Paul.

Carroll AR, Copp BR, Davis RA, Keyzers RA, Prinsep MR. 2019. Marine natural products. Nat Prod Rep 36: 122-173. DOI: 10.1039/c8np00092a.

Cohen E, Koch L, Thu KM, Rahamim Y, Aluma Y, Ilan M, Yarden O, Carmeli S. 2011. Novel terpenoids of the fungus Aspergillus insuetus isolated from the Mediterranean sponge Psammocinia sp. collected along the coast of Israel. Bioorg Med Chem 19 (22): 6587-6593. DOI: 10.1016/j.bmc.2011.05.045.

Gonçalves MFM, Santos L, Silva BMV, Abreu AC, Vicente TFL, Esteves AC, Alves A. 2019. Biodiversity of Penicillium species from marine environments in Portugal and description of Penicillium lusitanum sp. nov., a novel species isolated from seawater. Int J Syst Evol Microbiol 69 (10): 3014-3021. DOI: 10.1099/ijsem.0.003535.

Danovaro R, Pusceddu A. 2007. Biodiversity and ecosystem functioning in coastal lagoons: does microbial diversity play any role? Estuar Coast Shelf Sci 75 (1-2): 4-12. DOI: 10.1016/j.ecss.2007.02.030.
Das S, Lyla PS, Khan SA. 2006. Marine microbial diversity and ecology: importance and future perspectives. Curr Sci 90 (10): 1325-1335.

Deshmukh SK, Prakash V, Ranjan N. 2018. Marine fungi: a source of potential anticancer compounds. Front Microbiol 8: 2536. DOI: 10.3389/fmicb.2017.02536.

Holmström C, Kjelleberg S. 1999. Marine Pseudoalteromonas species are associated with higher organisms and produce biologically active extracellular agents. FEMS Microbiol Ecol 30 (4): 285-293. DOI: 10.1111/j.1574-6941.1999tb00656. x.

Justo A, Hibbett DS. 2011. Phylogenetic classification of Trametes (Basidiomycota, Polyporales) based on a five-marker dataset. Taxon 60 (6): 1567=1583. DOI: 10.1002/tax.606003.

Kaur A, Rogers KD, Swenson DE, Dowd PF, Wicklow DT, Gloer JB. 2014. Bioactive natural products from fungicolous Hawaiian isolates: secondary metabolites from a Phialemoniopsis sp. Mycology 5 (3): 120-129. DOI: 10.1080/21501203.2014.931309.

Khalmuratova I, Kim H, Nam YJ, Oh Y, Jeong MJ, Choi HR, You YH, Choo YS, Lee IJ, Shin JH, Yoon H, Kim JG. 2015. Diversity and plant growth-promoting capacity of endophytic fungi associated with halophytic plants from the west coast of Korea. Mycobiology 43 (4): 373-383. DOI: 10.5941/MYCO.2015.43.4.373.

Knežević A, Stajić M, Sofrenić I, Stanojković T, Milovanović I, Tešević V, Vukojević J. 2018. Antioxidative, antifungal, cytotoxic and antineurodegenerative activity of selected Trametes species from $\begin{array}{lllll}\text { Serbia. PloS One } 13 \text { (8): e0203064. DOI: } & \end{array}$ 10.1371/journal.pone.0203064.

Kumar S, Stecher G, Tamura K. 2016. MEGA7: molecular evolutionary genetics analysis version 7.0 for bigger datasets. Mol Biol Evol 33 (7): 1870-1874. DOI: 10.1093/molbev/msw054.

Leboffe MJ, Pierce BE. 2011. A photographic atlas for the microbiology laboratory ( $4^{\text {th }}$ edition). Morton Publishing, Colorado.

Li L, Singh P, Liu Y, Pan S, Wang G. 2014. Diversity and biochemical features of culturable fungi from the coastal waters of Southern China. AMB Express 4: 60. DOI: 10.1186/s13568-014-0060-9.

Mahanty S, Bakshi M, Ghosh S, Chatterjee S, Bhattacharyya S, Das P, Das S, Chaudhuri P. 2019. Green synthesis of iron oxide nanoparticles mediated by filamentous fungi isolated from Sundarban mangrove ecosystem, India. BioNanoScience 9: 637-651. DOI: 10.1007/s12668-019-00644-w.

Manohar CS, Raghukumar C. 2013. Fungal diversity from various marine habitats deduced through culture-independent studies. FEMS Microbiol Lett 341 (2): 69-78. DOI: 10.1111/1574-6968.12087.

Mathan S, Smith AA, Kumaran J, Prakash S. 2011. Anticancer and antimicrobial activity of Aspergillus protuberus SP1 isolated from marine sediments of South Indian coast. Chinese J Nat Med 9 (4): 286-292. DOI: 10.1016/S1875-5364(11)60066-6.

Migahed FF. 2003. Distribution of fungi in the sandy soil of Egyptian $\begin{array}{llll}\text { beaches. } & \text { Mycobiology } 31 & \text { (2): 61-67. DOI: }\end{array}$ 10.4489/MYCO.2003.31.2.061.

Perdomo H, Garcia D, Gene J, Cano J, Sutton DA, Summerbell R, Guarro J. 2013. Phialemoniopsis, a new genus of Sordariomycetes, and new species of Phialemonium and Lecythophora. Mycologia 105 (2): 398421. DOI: $10.3852 / 12-137$.

Qin QL, Li Y, Zhang YJ, Zhou ZM, Zhang WX, Chen XL, Zhang XY, Zhou BC, Wang L, Zhang YZ. 2011. Comparative genomics reveals a deep-sea sediment-adapted lifestyle of Pseudoalteromonas sp. SM9913. The ISME J 5 (2): 274-284. DOI: 10.1038/ismej.2010.103.

Richards GP, Needleman DS, Watson MA, Polson SW. 2019. Wholegenome sequences of two Pseudoalteromonas piscicida strains, DE1A and DE2-A, with strong antibacterial activity against Vibrio vulnificus. Microbiol Resour Announcements 8 (1): e01451-18. DOI: 10.1128/MRA.01451-18.

Rédou V, Navarri M, Meslet-Cladière L, Barbier G, Burgaud G. 2015. Species richness and adaptation of marine fungi from deepsubseafloor sediments. Appl Environ Microbiol 81 (10): 3571-3583. DOI: 10.1128/AEM.04064-14.

Siegenthaler A, Wangensteen OS, Benvenuto C, Campos J, Mariani S. 2018. DNA metabarcoding unveils large-scale trophic variation in a widespread coastal opportunist. Mol Ecol 28 (2): 232-249. DOI: 10.1111/mec.14886.

Taha H, Shivanand P, Shaminan NIN, Osman M, Abdul-Halim AMAA, Abdullah M. 2020a. Isolation and identification of culturable bacteria and fungi from mixed dipterocarp and mangrove forests of Brunei 
Darussalam. Proc Natl Acad Sci India Section B Biol Sci 90 (3): 523 530. DOI: $10.1007 / \mathrm{s} 40011-019-01119-4$.

Taha H, Shivanand P, Khoo DH, Mohammad YH, Matussin NBA, Metali F. 2020b. Identification of culturable petroleum-degrading bacteria and fungi from petroleum-contaminated sites in Brunei Darussalam. Environ Sci Health Part A 55 (13): 1542-1547. DOI: 10.1080/10934529.2020.1826238.

Yakop F, Taha H, Shivanand P. 2019. Isolation of fungi from various habitats and their possible bioremediation. Curr Sci 116 (5): 733-740. DOI: $10.18520 / \mathrm{cs} / \mathrm{v} 116 / 15 / 733-740$.
Yue Y, Yu H, Li R, Xing R, Liu S, Li P. 2015. Exploring the antibacterial and antifungal potential of jellyfish-associated marine fungi by cultivation-dependent approaches. PLoS ONE 10 (12): e0144394. DOI: 10.1371/journal.pone.0144394.

Zakaria L, Yee TL, Zakaria M, Salleh B. 2011. Diversity of microfungi in sandy beach soil of Teluk Aling, Pulau Pinang. Trop Life Sci Res 22 (1): 71-80.

Zhang L, An R, Wang J, Sun N, Zhang S, Hu J, Kuai J. 2005. Exploring novel bioactive compounds from marine microbes. Curr Opin Microbiol 8 (3): 276-281. DOI: 10.1016/j.mib.2005.04.008. 